\title{
A New Approach for Cervical Vertebrae Segmentation
}

\author{
Saïd Mahmoudi and Mohammed Benjelloun \\ Computer Science Department, Faculty of Engineering, \\ rue de Houdain 9 Mons, B-7000, Belgium \\ \{Said.Mahmoudi, Mohammed.Benjelloun\}@fpms.ac.be
}

\begin{abstract}
Efficient content-based image retrieval of biomedical images is a challenging problem of growing research interest. This paper describes how X-ray images of the spinal columns are analyzed in order to extract vertebra regions and contours. Our goal is to develop a computer vision tool able to determine a global polygonal region for each vertebra in first time. After this step, we apply a polar signature system in order to extract the effective contour of each vertebra. Finally, we use an edge closing method exploiting a polynomial fitting. The aim is to propose a closed contours detection representing each vertebra separately. We suggest an application of the proposed method which consists on an evaluation of vertebra motion induced by their movement between two or several positions.
\end{abstract}

Keywords: Vertebral Mobility Analysis, X-ray Images, Region Vertebra Selection, Contour Detection, Template Matching, Polar Signature.

\section{Introduction}

Medical staffs often examine X-rays of spinal columns to determine the presence of abnormalities or dysfunctions and to analyze the vertebral mobility. Nevertheless, the result is generally qualitative and subjective. To help them to establish a good diagnosis, medical image processing and analysis applications automate some tasks dealing with the interpretation of these images. It permits the extraction of quantitative and objective parameters related to the form and the texture included in pictures. These image parameters allow to measure, compare and detect the changes between images. X-ray images segmentation is an essential task for morphology analysis and motion estimation of the spinal column. Several methods have been proposed in the literature to analyze and to extract vertebra contours from X-ray images [1]. Extensive research has been done by Long et al. [19] to automatically identify and classify spinal vertebrae. They formulated the problem of spine vertebra identification by three level of processing: In the first stage they used an heuristic analysis combined with an adaptive thresholding system to obtain basic orientation data, providing basic landmarks in the image; in the second stage, boundary data for the spine region of interest were defined by solving an optimization problem; the third stage was expected 
to use deformable template processing to locate individual vertebra boundaries at finely grained level. The main drawback of this approach is the need of a good grayscale thresholding. Stanley and Long [2] proposed a new method of subluxation detection. They used the spatial location of each vertebra in the spinal column and the variation in its position. They applied a second order spinal column approximation by using vertebral centroids. The goal of their approach was to quantify the degree to which vertebra areas within the image are positioned on their posterior sides.

In an other work, Kauffmann et al. 4] first detected the axis of the spinal column by manually placing points along it and fitting a curve through them. The fitted curve was used to initialize and rigidly match templates of vertebral body with the image data to obtain vertebral outlines. Verdonck et al. 5] manually indicated specific landmarks in the image and founded others using an interpolation technique. The landmarks, together with a manually indicated axis of the spinal column, were used to automatically compute endplates on vertebrae and the global outline of the spine.

Techniques using Hough transform 667] and Active Shape Models [8] are other examples of the various approaches developed. These methods use a large set of templates to capture the great variability in vertebra shapes. But, in most of the cases, it leads to prohibitive computation time, as in the case of Hough transform, and usually needs a large and accurate training set in the case of Active Shape Models.

In this work, we propose a new segmentation approach applied to vertebral mobility analysis. The proposed segmentation approach is based on a first semiautomatic step of region vertebra selection. After this, we achieve vertebra contour detection using a polar signature system followed by a polynomial fitting process. The extraction of some quantitative measures of particular changes between images acquired at different moments allows determining vertebral mobility. For instance, to measure and compare the corresponding vertebrae between several images, we analyze vertebra edges extracted from some images corresponding to the cervical vertebra of the same person, in flexion, neutral and extension positions. This paper is organized as follows: In section 2 we present the principles of the region vertebra selection process. After this we describe in section 3 the polar signature system used for vertebra contours detection. In section 4, we describe the numerical segmentation results given by this method, and presented by the angular measures of each vertebra. These results allow the estimation of vertebral mobility.

\section{Region Vertebra Selection}

This first step allows the creation of a polygonal region for each vertebra. This will facilitate edge detection, and also make easier other processing like determining relative positions between vertebrae. Each region represents a specific geometrical model based on the geometry and the orientation of each vertebra. We propose a process where the user has to click once inside each vertebra. 
We initially place a click by vertebra, towards the center of the vertebra. These landmarks represent the starting points $P\left(x_{i}, y_{i}\right)$ for the construction of vertebra regions, figure (1 $1 \mathrm{a}$ ). After this, we compute the distance between each two contiguous points $\left(D_{i, i+1}\right)$, equation (1), and the line $L_{1}$, figure (1) b), which connects the contiguous points, by a first order polynomial, equation (2).

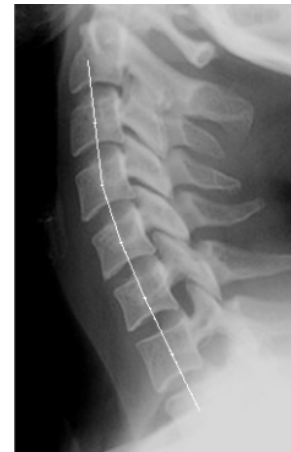

(a)

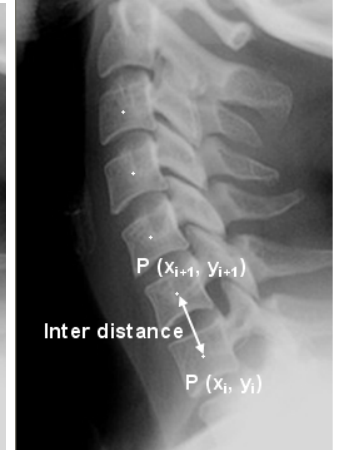

(b)

Fig. 1. (a) : The first order polynomial function: $\left(L_{1}\right)$ between the click points. (b): The inter vertebral distance computation, $P\left(x_{i}, y_{i}\right)$ and $P\left(x_{i+1}, y_{i+1}\right)$ are the click points associated to the first and the second vertebrae.

$$
\begin{gathered}
D_{i, i+1}=\sqrt{\left(P\left(x_{i}, y_{i}\right)-P\left(x_{i+1}, y_{i+1}\right)\right)^{2}} \\
L_{1}=f\left[a, b ; P\left(x_{i}, y_{i}\right), P\left(x_{i+1}, y_{i+1}\right)\right]
\end{gathered}
$$

The calculated distance between the click points allows the estimation of the inter vertebral distance. We use the line $L_{1}$ and the consecutive click points to carry out a relative estimation of order zero for the angles between vertebrae.

On the other hand, the line $L_{1}$ will be used as reference for a template displacement by the function $T(x, y)$. This template function represents an intervertebral model, which is calculated according to the area shapes between vertebrae. To build the function $T(x, y)$, we analyzed the figures (2 a) and (2-b). The figure (2-b) presents the intensity values distribution along the line $L_{1}$. We notice that this intensity deployment can be decomposed of two main shapes: vertebral and inter-vertebral areas. We focus our analysis on the inter-vertebral area which takes a valley form. So, we propose a template function $T(x, y)$ with an opposite exponential trajectory. This function is given by equation (3). This template function depends on a new reference plane on the direction of $L_{1}$. Figure $(2 \mathrm{r})$ is a three dimensional representation of the template function $T(x, y)$ which is built by auto repetition of $T(x, y)$ along an axis.

We use the $L_{1}$ function and the inter vertebral distances to calculate the inter vertebral angles $\left(\alpha_{i v}\right)$ and to determine a division line for each inter vertebral area. The goal of the proposed template matching process is to find the position 


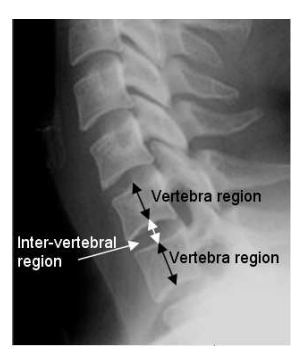

(a)

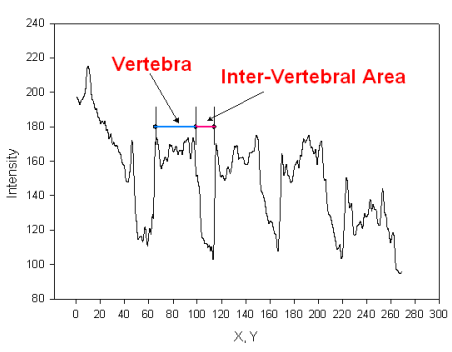

(b)

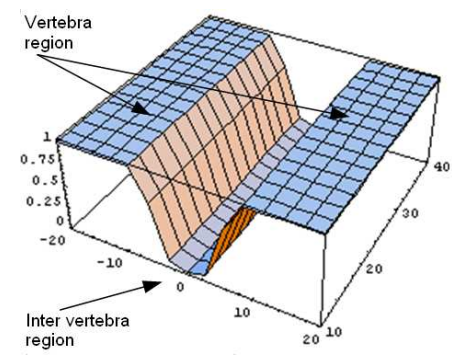

(c)

Fig. 2. (a): Representation of the vertebra area and the inter vertebra region; (b): Distribution of the of intensity values along the line $L_{1}$; (c): Three dimensional representation of the template function proposed

on the image which is best correlated with the template function (See [3] for more detail). So, for each vertebra, the template function $T(x, y)$ is first placed on the geometrical inter-vertebral central point $P\left(x_{i c}, y_{i c}\right)$, which represents the average position between each two contiguous click points: $P\left(x_{i}, y_{i}\right)$ and $P\left(x_{i+1}, y_{i+1}\right)$. The new reference plane is created with $P\left(x_{i c}, y_{i c}\right)$ as center. The $X$ axis of this plane is the line $L_{1}$. The $Y$ is axis therefore easily created by tracing the line passing through $P\left(x_{i c}, y_{i c}\right)$ and orthogonal to $L_{1}$. We notice that the orientation angle of this second axis present the initial value of the orientation angle $\alpha_{i v}$. To determine the points representing border's areas, we displace the template function $T(x, y)$, equation (3) , between each two reference points $P\left(x_{i}, y_{i}\right)$ and $P\left(x_{i+1}, y_{i+1}\right)$, along the line $L_{1}$. Then, we compute the correlation degree $D_{C}$ between the template function and the image $I(x, y)$. The central geometrical point is moved in the two directions in top and bottom along the $L_{1}$ axis for a distance equivalent to a parameter $T_{r}$ fixed experimentally according to the X-ray images used at $\pm 25 \%$ of the inter-vertebral distance $\left(D_{i, i+1}\right)$. Also, for each position on the line $L_{1}$ obtained by this translation, we operate a shift of the orientation angle $\alpha_{i v}$ using an angle parameter $\beta_{r}$, fixed experimentally at $\pm 30^{\circ},\left(T(x, y) \pm \beta_{r}\right)$, with a step of $2^{\circ}$.

$$
T(x, y)=\left(1-e^{\left(-r x_{l}^{2}\right)}\right) \quad \text { with } \quad r=k / D_{i, i+1}
$$

With $k=0.1$ an empirical value and $x_{l}$ the coordinate of the point $(x, y)$ in the new reference plane.

The correlation degree is a similarity measurement which permits to obtain the ideal template function that joins perfectly the borders between the areas of vertebrae. The maximum correlation value $D_{C}$ between templates function $T(x, y)$ and the image $I(x, y)$ for all the analyzed positions will correspond to the most stable position. This position corresponds to an angle $\alpha_{i v}$ and a position $P\left(x_{i M}, y_{i M}\right)$ for the template function, i.e. the position on the image $\left(P\left(x_{i}, y_{i}\right) \pm T_{r} * D_{(i, i+1)}, \pm \beta_{r}\right)$ in which the template function $T(x, y)$ is best placed. In figure (3.a), the click points $P\left(x_{i}, y_{i}\right)$ are represented. In figure (3-b), 
we present the inter-vertebral points $P\left(x_{i M}, y_{i M}\right)$ given by the proposed procedure. In figure (3. $)$, boundary lines between vertebrae are traced according to the angle $\alpha_{i v}$ given by the same procedure and centered on the points $\left.P\left(x_{i M}, y_{i M}\right)\right)$. To obtain vertebral regions, we connect the extreme points of the boundary lines, figure (3rd).

The Results obtained by the region vertebra segmentation method in flexion and neutral and extension positions are represented in figure 4. Already at this step, we can estimate vertebral mobility. Indeed, the mobility of the vertebrae can be approximated by the mobility of their anterior sides. We rely on angular variations measurements and comparisons to determine this one. Once all the segments that represent each polygonal region are found, we extract the segments representing the anterior (frontal) faces (3]). After this, we can make a first estimation of the orientation angle belonging to each vertebra, the angular variation between two consecutive vertebrae and the angular variation for the same vertebra in two different positions. But, to have better precision, we apply the contour detection of each vertebra inside its area. This process allows the computation of some parameters characterizing each vertebra, like their positions, dimensions, orientation, and other cervical information.

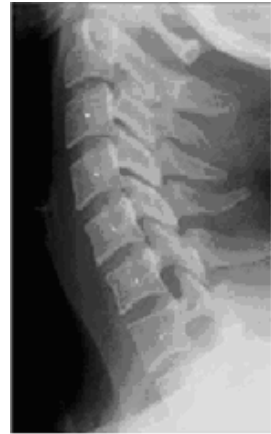

(a) original image reference

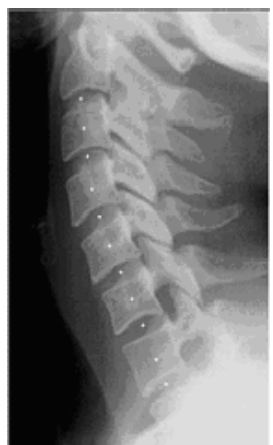

(b) order points given by template matching process

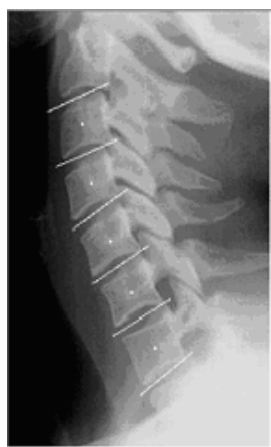

(c) boundary lines between vertebrae

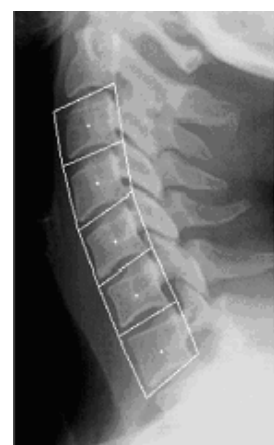

(d) vertebrae regions

Fig. 3. Results obtained by the region vertebra segmentation method

\section{Contour Detection}

After the region localization step, we proceed to vertebra contours detection. To this aim, we use a polar signature method 12 applied to each vertebra region. A general approach to determine the polar signature of objects boundaries is illustrated in figure (6). We choose to use this polar signature approach in order to explore all region points likely to be corresponding to vertebra contours.

A polar signature technique applied to vertebra region is represented in figure (7). The center point of the polar signature system is the click point. We 


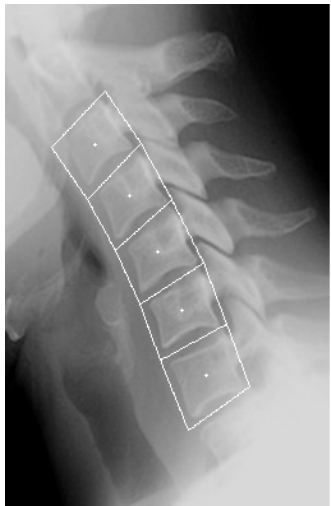

(a)

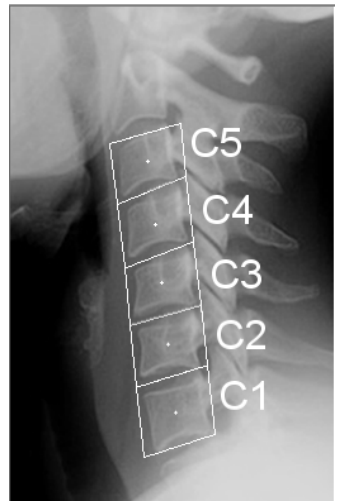

(b)

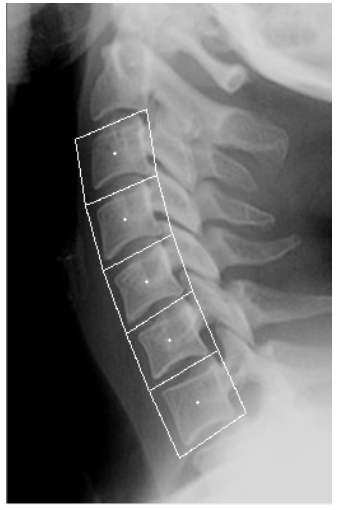

(c)

Fig. 4. Results obtained by the region vertebra segmentation method for three X-ray images of the spinal column in three positions: (a): flexion position, (b): reference position, (c): extension position

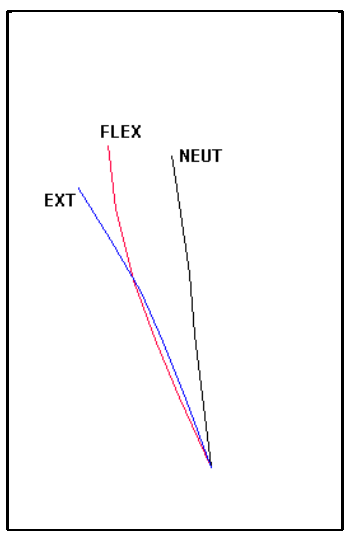

Fig. 5. Graphical comparison between three cervical spine positions using a frontal face computing by Region vertebra segmentation

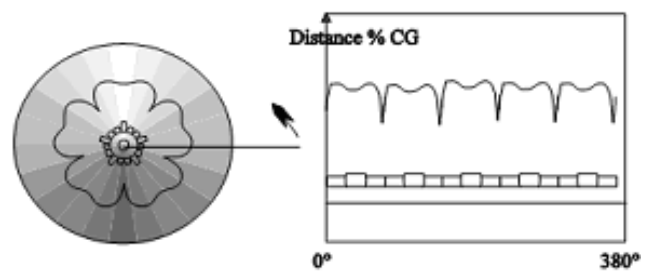

Fig. 6. Example of contour points obtained by a polar signature approach 
make the radial vector turn by $360^{\circ}$ around the central points with a step parameter, $\Delta_{\alpha}$ degree. More $\Delta_{\alpha}$ is high, more the computing time is less. But more $\Delta_{\alpha}$ is high, more the contour is open. In order to get a closed contour, we apply an edge closing method to the contours obtained, a polynomial fitting to each face for each vertebra. In deed, for a better approximation of vertebra contours, we use a second degree polynomial fitting [10]. We achieve this $2 \mathrm{D}$ polynomial fitting by the least square method, figures (8 $\mathrm{r}$ a, 8 $\mathrm{rb}$ ).
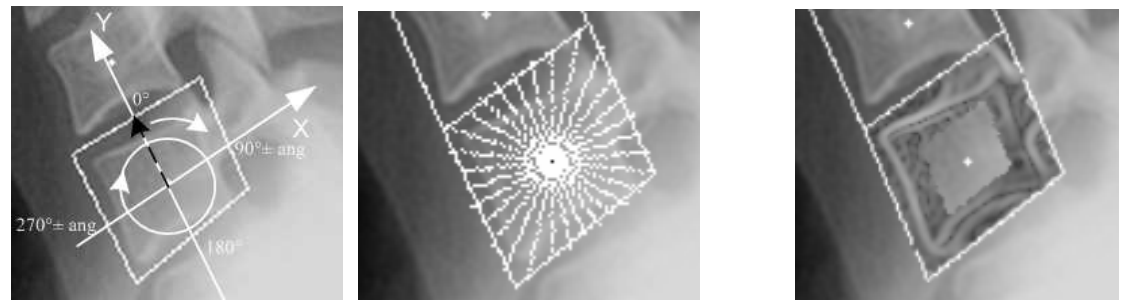

(a) Polar signature system (b) Polar signature direction (c) Research area in Gradient intensity

Fig. 7. Polar signature applied to vertebra region
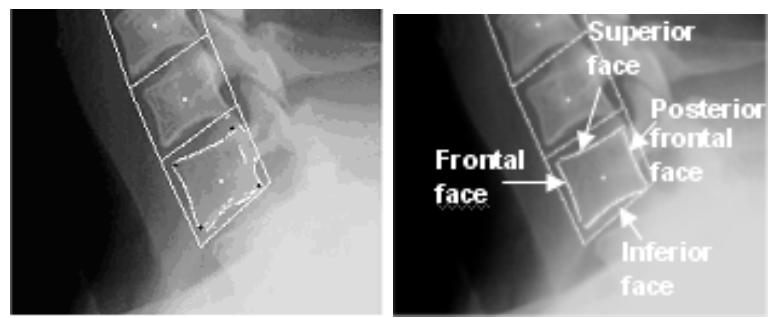

Fig. 8. (a) Contour and corners points, (b) Polynomial fitting for each vertebra face

\section{Experimental Results}

We apply the proposed method to a large set of X-ray images of the cervical spinal column. We have tested the algorithm on a set of 100 images belonging to real patients. The figure (9). shows the results obtained by applying the proposed method to three X-ray images of the cervical spinal column. We notice that the process of region selection, figure (3) gives good results and permits to isolate each vertebra separately in a polygonal area. On the other hand, contours extracted with the polar signature system combined with template matching process are given with high precision. The great advantage of our method is the fact that segmentation results are presented by closed contours. This will essentially facilitate the use of these results for image indexing and retrieval. 


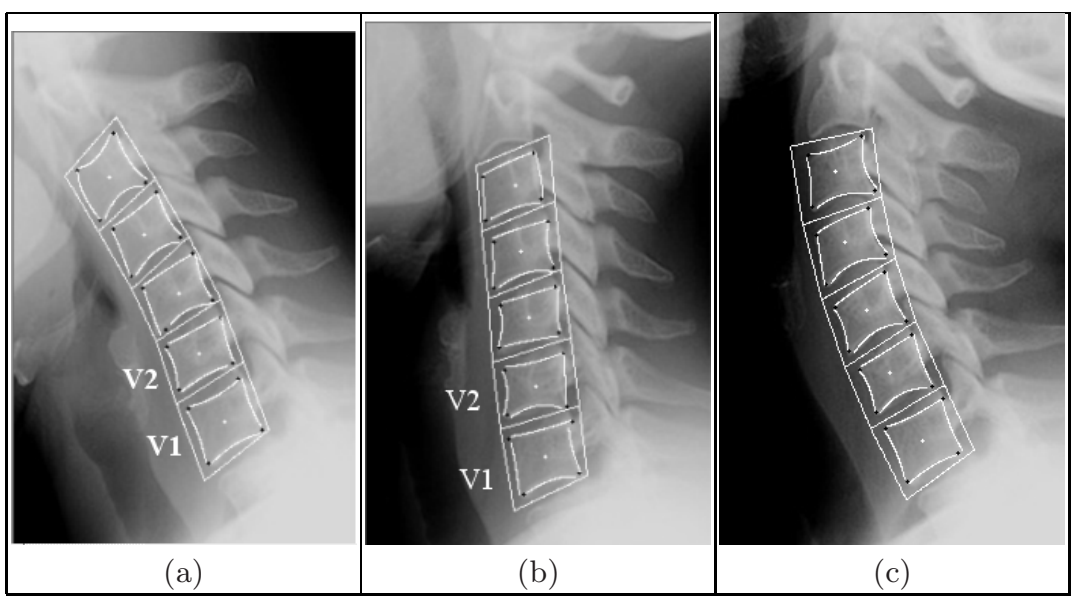

Fig. 9. Results obtained by the proposed contour vertebra selection method

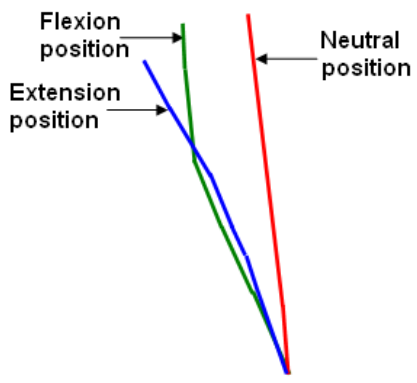

Fig. 10. Graphical comparison between three spine positions

Vertebral mobility is estimated by the computation of the orientation angles belonging to each face of the contour. In table 1, we present some quantitative measurements of the orientation angle for each vertebra face. So, we present the orientation angles for the five vertebrae belonging to the images (a), (b) and (c) in figure (9). This allows motion head estimation and vertebral mobility computation. The error margin by applying only region segmentation method instead of the region vertebra segmentation combined with polar signature is shown in table (2). The results given in table (2) are corresponding to the neutral position and the frontal faces of vertebrae $V 1$ to $V 5$. Two graphical representation of the angular variation between these three positions using region and contour detection with polar signature methods are shown in figures (5) and (10). It will be noticed that if figures (5) and (10) resemble, the error margin Delta is rather significant. This shows the utility of an additional treatment like the polar signature. 
Table 1. Orientation angles for the five first vetebrae in figure. 9, with $S_{p}=1^{\circ}$

\begin{tabular}{|c|c|c|c|c|c|c|c|c|c|c|c|c|c|c|c|c|c|}
\hline \multicolumn{4}{|c|}{ PF: Posterior face } & \multicolumn{11}{|c|}{\begin{tabular}{l|l|l|} 
FF: Frontal face & SF: Superior face & IF: Inferior face \\
\end{tabular}} & \multirow[b]{2}{*}{$\overline{F F}$} & \multirow{2}{*}{\multicolumn{2}{|c|}{\begin{tabular}{|l|l|} 
SF & IF \\
\end{tabular}}} \\
\hline & & $\overline{\mathbf{P F}}$ & $\overline{\text { FF }}$ & SF & IF & & & $\overline{\mathbf{P F}}$ & $\overline{F F}$ & SF & IF & & & $\overline{\overline{\text { PF }}}$ & & & \\
\hline \multirow{5}{*}{ (a) } & $V_{1}$ & 114.0 & 113.6 & 25.3 & 29.3 & & $V_{1}$ & 103.8 & 102.0 & 15.6 & 18.5 & & $V_{1}$ & 117.0 & 113.5 & 26.9 & 29.8 \\
\hline & $V_{2}$ & 107.7 & 106.4 & 26.8 & 25.5 & (b) & $V_{2}$ & 93.1 & 91.7 & 13.1 & 8.6 & (c) & $V_{2}$ & 111.5 & 110.5 & 12.7 & 28.2 \\
\hline & $V_{3}$ & 112.0 & 110.0 & 32.4 & 29.7 & & $V_{3}$ & 101.1 & 90.0 & 15.2 & 8.1 & & 3 & 112.6 & 106.2 & 34.0 & 31.4 \\
\hline & $V_{4}$ & 119.5 & \begin{tabular}{|l}
13.8 \\
\end{tabular} & 37.2 & 37.3 & & $V_{4}$ & 102.1 & 91.6 & 16.4 & 14.3 & & $V_{4}$ & 112.5 & 99.4 & 23.2 & 24.5 \\
\hline & $V_{5}$ & 124.6 & 127.6 & 49.4 & 43.0 & & $V_{5}$ & 104.3 & 91.7 & 23.5 & 17.9 & & $V_{5}$ & 107.1 & 90.0 & 18.8 & 13.3 \\
\hline
\end{tabular}

Table 2. Error margin between (R.V): frontal face detection with only region vertebra selection method and (S.P): frontal face detection with polar signature approach

\begin{tabular}{|c|c|c|}
\hline R.V + S.P. & R.V. & Delta \\
\hline 101,6 & 99 & $101,6-99=2,6$ \\
\hline 91,7 & 95,9 & $-4,2$ \\
\hline 90 & 94,9 & $-4,9$ \\
\hline 91,6 & 98,3 & $-6,7$ \\
\hline 91,7 & 98,1 & $-6,4$ \\
\hline
\end{tabular}

\section{Conclusion}

In this a paper, a new method of vertebra segmentation has been proposed. The goal of this work was to propose a method for closed contours detection representing each vertebra separately. This method permits to overcome some classical problems related to closed contours extraction. Our approach lies on three steps. First, we proposed a region vertebra selection. This step allows the creation of a polygonal region for each vertebra and facilitate edge detection. In the second step we applied a polar signature to extract the effective contour of each vertebra. Finally, we used an edge closing method exploiting a polynomial fitting. We have applied, with good results, the method to a large set of real images. The major advantage of the polar signature is the facility and the precision of the results. But, if the precision is obtained by increasing azimuths number, the cost in time computing can be sometimes unfavorable according to images complexity. After the contour segmentation process, we have estimated vertebral mobility. The applied techniques have given good results to measure the mobility of cervical vertebrae. For this, we calculated the angular variations between two consecutive vertebrae within the same image as well to measure the angular variation of a vertebra in several images, in particular between three cervical spine positions. In our future works, we are aiming to develop a template matching method for all the process of contour extraction, and also limiting to only one the number of click points initially placed by the user. Currently we are developing a content based image retrieval system by using the results presented in this paper. 


\section{References}

1. Long, L.R., Thoma, G.R.: Identification and classification of spine vertebrae by automated methods. SPIE Medical Imaging 2001 Image Processing, vol. 4322 (2001)

2. Stanley, R.J, Seetharaman, S., Long, R.L., Antani, S., Thoma, G.: Downey Ed. image analysis techniques for the automated evaluation of subaxial subluxation in cervical spine x-ray images. In: Proceeding of the 17th IEEE symposium on computer-based medical systems CMBS 2004 (2004)

3. Benjelloun, M., Téllez, H., Mahmoudi, S.: Template matching method for vertebra region selection. In: ICTTA 2006, Damascus, Syria (April 2006)

4. Kauffman, C., Guise, J.: Digital radiography segmentation of scoliotic vertebral body using deformable models. SPIE Medical Imaging vol 3034, pp. 243-251 (1997)

5. Verdonck, B., Nijlunsing, R., Gerritsenand, F., Cheung, J., Wever, D., Veldhuizen, A., Devillers, S., Makram-Ebeid, S.: Computer. In: Proceeding of International Conference of Computing and Computer Assisted Interventions. LNCS, pp. 822 831. Springer, Heidelberg (1998)

6. Howe, B., Gururajan, A., Sari-Sarraf, H., Long, R.: Hierarchical Segmentation of Cervical and Lumbar Vertebrae Using a Customized Generalized Hough Transform. In: Proc. IEEE 6th SSIAI, p. 182-186, Lake Tahoe, NV, March (2004)

7. Tezmol, A., Sari-Sarraf, H., Mitra, S., Long, R., Gururajan, A.: A Customized Hough Transform for Robust Segmentation of Cervical Vertebrae from X-Ray Images. In: Proc. 5th IEEE Southwest Symposium on Image Analysis and Interpretation, santa Fe, NMexico, USA (2002)

8. Roberts, M.G., Cootes, T.F., Adams, J.E.: Linking Sequences of Active Appearance Sub-Models via Constraints: an Application in Automated Vertebral Morphometry. In: Proc. BMVC 2003, vol.1, pp. 349-358 (2003)

9. Long, L.R., Thoma, G.R.: Use of shape models to search digitized spine X-rays. In: Proc. IEEE Computer-Based Medical Systems, Houston, TX, pp. 255-260 (June 2000)

10. Keren, D.: Topologically Faithful Fitting of Simple Closed Curves. IEEE Transactions on PAMI 26(1) (2004)

11. Rico, G., Benjelloun, M., Libert, G.: Detection, Localization and Representation of Cervical Vertebrae. In: Computer Vision Winter Workshop 2001; Bled, Slovenia; pp. 114-124 (February 2001)

12. Lie, W.N., Chen, Y.C.: Shape representation and matching using polar signature. In: Proc. Intl. Comput. Symp. 1986, pp. 710-718 (1986) 